\title{
Anni Borzeix, Gwenaëlle Rot, Sociologie du travail. Genèse d'une discipline, naissance d'une revue
}

\section{Gérald Houdeville}

\section{OpenEdition}

\section{Journals}

Édition électronique

URL : http://journals.openedition.org/travailemploi/5271

DOI : 10.4000/travailemploi.5271

ISSN : 1775-416X

Éditeur

DARES - Ministère du Travail

\section{Édition imprimée}

Date de publication : 15 juin 2011

Pagination : 74-75

ISSN : 0224-4365

\section{Référence électronique}

Gérald Houdeville, « Anni Borzeix, Gwenaëlle Rot, Sociologie du travail. Genèse d'une discipline, naissance d'une revue », Travail et Emploi [En ligne], 126 | avril-juin 2011, mis en ligne le 24 février 2012, consulté le 22 septembre 2020. URL : http://journals.openedition.org/travailemploi/5271 ; DOI : https://doi.org/ 10.4000/travailemploi.5271

Ce document a été généré automatiquement le 22 septembre 2020.

(c) Direction de l'animation de la recherche, des études et des statistiques (Dares) 


\title{
Anni Borzeix, Gwenaëlle Rot, Sociologie du travail. Genèse d'une discipline, naissance d'une revue
}

\author{
Gérald Houdeville
}

\section{RÉFÉRENCE}

Anni Borzeix, Gwenaëlle Rot, Sociologie du travail. Genèse d'une discipline, naissance d'une revue, Paris, Presses universitaires de Paris Ouest, 2010.

1 L'ouvrage s'efforce de cerner les conditions de la création, en 1959, de la revue Sociologie du travail et de la production des vingt-cinq premiers numéros (1959/1966) de cette revue parvenue jusqu'à aujourd'hui à préserver son statut de référence en sociologie. Elle est destinée, à l'époque, à faire connaître les perspectives sociologiques à « un large public : chefs d'entreprises, syndicalistes, organisateurs, universitaires [...] qui cherchent à comprendre les problèmes du travail ». Le projet du livre est toutefois plus large puisqu'à travers l'examen des conditions d'apparition et des premières années d'activité de la revue, il s'agit de dégager «le portrait de la communauté socioprofessionnelle » de la sociologie du travail en France et, plus encore, de porter au jour les «fondements intellectuels d'une discipline et des conditions institutionnelles de production de la sociologie ».

2 Ce faisant, les auteurs exploitent un sillon signalé par d'autres ${ }^{1}$, il y a déjà vingt ans, comme fécond : l'examen des premières recherches de sociologie du travail réalisée en France après 1945 comme creuset des démarches devenues par la suite typiques de la sociologie en général en matière de traitement des données produites par des organismes d'État et/ou issues d'un travail de première main réalisé dans le cadre d'enquêtes de terrain" (observation directe, entretiens, questionnaires) par les sociologues eux-mêmes. Parce qu'elle porte son attention sur un corpus clairement circonscrit et limité, l'approche des deux auteurs se révèle intensive et très 
circonstanciée: elles soulignent, à la fois, le caractère déterminant de l'action d'individualités - notamment la grande figure de Georges Friedmann, l'auteur des "Problèmes humains du machinisme industriel" en 1947 - sans lequel la suite, en matière d'organisation de l'activité de recherche, est proprement inconcevable, et portent sur le devant de la scène, par un effet de focale, l'activité décisive de collectifs de recherche plus nombreux qu'il n'apparaîtrait à un regard plus lointain porté sur cette période. Le choix de placer l'échelle de leur analyse des conditions d'émergence et de structuration de la sociologie au niveau d'un examen des conditions de la création et de l'activité d'une revue distingue leur entreprise de celle d'autres chercheurs.On pense notamment au précédent travail de Gwenaëlle Rot ayant cherché à éclairer le même phénomène à partir d'investigations centrées sur l'atelier et fondées sur un balayage des catégories de travaux ayant prévalu en la matière des années d'après-guerre aux années $2000^{2}$. On pourrait citer également les recherches de Lucie Tanguy portant, non pas tant sur la constitution de la sociologie en général (ses formes, ses caractères dans tel ou tel pays), que sur la constitution de la sociologie du travail en France en tant que spécialité, basées cette fois sur l'analyse d'archives du ministère du Travail - pour ce qui concerne la période de l'immédiat après-guerre ${ }^{3}$. Les analyses de Borzeix et Rot, quant à elles, sont construites sur des entretiens auprès des responsables éditoriaux de la revue au cours de la période considérée (Michel Crozier, Jean-Daniel Reynaud, Alain Touraine, Jean-René Tréanton), des archives issues de fonds distincts et, enfin, des travaux de recherche relatifs au contexte des activités sociologiques au cours des années 1950 et 1960.

3 La première partie de l'ouvrage permet de documenter les aspects relatifs à l'identité et à la trajectoire des quatre fondateurs, "jeunes intellectuels de bonne famille ", et, surtout, à l'univers dans lequel leur rencontre va s'opérer alors qu'ils ont autour de 35 ans à l'époque. Ce faisant, le livre permet un accès aux catégories de perception et d'action qui étaient celles de ces hommes - en tout cas telles qu'elles transparaissent à travers leur point de vue à ce sujet reconstruit quelques décennies plus tard à l'occasion des entretiens que les auteurs du livre ont réalisé auprès d'eux - et au tissu de relations dans lequel ils étaient insérés : formés à l'Ecole Normale Supérieure (ENS) et agrégés pour deux d'entre eux, diplômés de sciences politiques et des Hautes Etudes Commerciales (HEC) pour les deux autres, ils fréquentent les mêmes cercles parisiens d'une activité de recherche en sociologie en voie de structuration, à savoir le Centre d'Etudes Sociologiques (CES) du CNRS et les séminaires de Georges Friedmann à l'Institut des Sciences Sociales du Travail (ISST). Ils font des séjours d'études aux ÉtatsUnis et conduisent plusieurs travaux d'enquête de terrain en tant que représentants d'une génération de sociologues convertie à l'empirie. Enfin, ils se retrouvent sur un positionnement politique commun de type réformiste, critique en tout cas vis-à-vis du « marxisme idéologique».

$4 \mathrm{Vu}$ d'aujourd'hui la revue apparaît comme un début où tout était encore à faire, à construire, où il n'y avait pas d'institution pouvant tenir lieu de structure organisant l'activité de recherche sociologique. Or, ce que montrent les deux auteurs, dans la deuxième partie de l'ouvrage, c'est que la création de la revue est tout autant une forme de premier aboutissement dans l'itinéraire de ceux qui sont amenés, les premiers, à en assurer la responsabilité éditoriale. Le crédit accordé au quatuor à prendre en main une nouvelle revue dans l'intrication des relations des éditions du Seuil et de l'ISST en témoigne. Les quatre peuvent faire valoir plusieurs années de pratiques sociologiques et la participation à de grosses enquêtes et, en toute légitimité 
alors, pouvoir prétendre au rôle de successeurs des "patrons" de la génération précédente des Friedmann, Jean Stœtzel et autre Georges Gurvitch. Lelivre s'attarde longuement et de façon très minutieuse, par exemple, sur l'enquête de « Mont-SaintMartin » réalisée dans l'agglomération de Longwy (novembre 1954/mai 1957) et relative aux "attitudes des ouvriers de la sidérurgie en face des changements techniques ", Reynaud et Touraine tenant le rôle de superviseur dans cette enquête présentée comme " pionnière [...], la première recherche collective réalisée au sein de l'ISST». Le livre revient également sur la contribution très active de nos quatre hommes aux entreprises éditoriales structurantes de l'époque en sociologie dont les projets courent tout au long de la deuxième partie des années 1950: le Traité de sociologie de Gurvitch, le Traité de sociologie du travail de Friedmann et Pierre Naville.

5 La troisième partie restitue l'aspect émergeant de "la communauté intellectuelle " dans laquelle la revue va trouver sa place et, en même temps, qu'au fil des numéros, Sociologie du travail va contribuer à structurer en ouvrant un espace propre de publication «aux questions relatives à ce domaine spécifique couvert par le terme générique de travail » lui-même ouvert à différents contextes nationaux. Le caractère émergeant de cette communauté intellectuelle autour de la sociologie du travail se "lit", en premier lieu, à travers l'identité des contributeurs « qui [certes] intègrent des questionnements sociologiques à leur démarche » mais sans que tous soient estampillés sociologues à strictement parler. Par ailleurs, dans ces premiers temps, la revue accueille d'abord les comptes-rendus des enquêtes conduites dans le cadre du CES ou de l'ISST et, bientôt, des "labos" qui se montent et s'organisent à l'exemple du Laboratoire de sociologie industrielle de Touraine ou bien du Centre de sociologie européenne de Raymond Aron, codirigé et animé par Pierre Bourdieu. Elle se fait, en outre, le relais de travaux de thèses, à l'exemple de ceux de Bernard Mottez, premier enquêteur associé à Jacques Dofny dans l'enquête de Mont-Saint-Martin, de Maurice Montuclard, qui va fonder, avec François Sellier, le Laboratoire d'économie et de sociologie du travail (LEST) à Aix-en-Provence au début des années 1960, etc. La revue, enfin, reste ouverte aux contributions de « la société civile » : au moment de sa conception, dans le cadre de l'ISST, la revue est annoncée comme accessible aux praticiens et fait effectivement appel à des cadres d'entreprise, "familiers du séminaire de Friedmann [ou bien] passeurs qui ont rendu possible la mise en œuvre d'enquêtes de sociologie industrielle». Les liens noués "dans des lieux comme le Club Jean Moulin», notamment par Crozier, peuvent expliquer la proximité qui est également recherchée mais aussi discutée, interrogée - entre la recherche et l'action publique (les représentants de l'État-planificateur) : il s'agit d' "éviter d'enfermer la sociologie du travail dans un espace trop académique ».

6 Cet aspect émergeant d'un espace propre à la recherche sociologique relative au travail s'accompagne pour autant, dès cette époque, de la revendication à porter «une certaine définition de la sociologie et du métier de sociologue ». On la voit à l'œuvre dans les comptes rendus de travaux d'enquêtes menés par leurs auteurs ( «monographies étayées par un dispositif de mesure des données et illustrées par des tableaux statistiques») ou bien encore dans les comptes-rendus d'ouvrages qui pointent sans ménagement les défauts majeurs : «la tentation philosophique, l'absence d'hypothèses et de principes d'analyse, l'absence de rigueur méthodologique et d'enquête systématique, le poids de l'idéologie». 
7 L'ouvrage prend le parti de traiter de la sociologie comme d'un ensemble d'activités pratiques, comme d'une activité de travail parmi d'autres en somme, et non seulement comme d'un ensemble d'idées. Il apporte ainsi une contribution déterminante à la compréhension de la définition de ces activités qu'on appelle "sociologie" : il parvient notamment à rendre sensible au rôle de catalyseur qu'ont pu jouer les premiers contributeurs de Sociologie du travail dans cette définition.

\section{NOTES}

1. Jean-Michel Chapoulie, "La seconde fondation de la sociologie française, les États-Unis et la classe ouvrière ", Revue Française de sociologie, vol. XXXII, 1991, p.321-364.

2. Gwenaële Rot, Sociologie de l'atelier. Renault, le travail ouvrier et le sociologue, Toulouse, Octarès, 2006.

3. Lucie Tanguy, La sociologie du travail en France. Enquête sur le travail des sociologues, 1950-1990, Paris, La découverte, 2011.

\section{AUTEURS}

\section{GÉRALD HOUDEVILLE}

Maître de conférences en sociologie, IPSA-UCO (Angers) CENS-Université Nantes 